Canad. Math. Bull. Vol. 20 (1), 1977

\title{
CONJUGACY OF ELEMENTS IN A NORMAL RING
}

\author{
TAW-PIN LIM
}

Let $\left(R,{ }^{*}\right)$ be a ring $R$ with an involution ${ }^{*}$, i.e., ${ }^{*}$ is a map $R \rightarrow R$ such that for all $a, b \in R$

$$
\begin{aligned}
(a+b)^{*} & =a^{*}+b^{*} \\
(a b)^{*} & =b^{*} a^{*} \\
a^{* *} & =a .
\end{aligned}
$$

The trace and norm of an element $a$ in $\left(R,{ }^{*}\right)$ are respectively

$$
T(a)=a+a^{*}, \quad N(a)=a a^{*} .
$$

$(R, *)$ is said to be a normal ring if for all $a \in R$

or equivalently,

$$
N(a)=N\left(a^{*}\right)
$$

$$
a a^{*}=a^{*} a .
$$

It is well-known that two real quaternionic elements $a$ and $b$ have the same trace and norm if and only if they are conjugates, i.e., there exists a non-zero quaternion $x$ such that $x a=b x$. This result is now extended to a normal ring $\left(R,{ }^{*}\right), R$ being not commutative and having no zero divisors.

As usual, we write $[x, y]=x y-y x$ for all $x, y \in R$. The symbol $Z$ denotes the center of $R$. Clearly, $x \in Z$ implies $x^{*} \in Z$.

Following Dyson [1], a ring $\left(R,{ }^{*}\right)$ is said to have the scalar product property (and is henceforth abbreviated as a SPP-ring) if for all $a, b \in R$

$$
\left[a^{*}, b^{*}\right]=[a, b]
$$

or equivalently,

$$
T(a b)=T(b a) .
$$

Lemma 1. (i) A normal ring $\left(R,{ }^{*}\right)$ is a SPP-ring.

(ii) A 2-torsionfree SSP-ring $\left(R,{ }^{*}\right)$ is a normal ring.

Proof. (i) For all $a, b \in R$

$$
\begin{aligned}
T(a b) & =N\left(a+b^{*}\right)-N(a)-N\left(b^{*}\right) \\
& =N\left(a^{*}+b\right)-N\left(a^{*}\right)-N(b) \\
& =T(b a) .
\end{aligned}
$$

Received by the editors Sept. 15, 1976. 
(ii) For all $a \in R, 2 a a^{*}=T\left(a a^{*}\right)=T\left(a^{*} a\right)=2 a^{*} a$. Hence, $a a^{*}=a^{*} a$.

A SPP-ring which is not 2-torsionfree need not be normal. We have the following

EXAMPLE 1. Let $F$ be a field of char 2 and $R$ be the $F$-algebra of matrices of the form:

$$
\left[\begin{array}{ccc}
x & y & z \\
0 & x & w \\
0 & 0 & x
\end{array}\right], \quad x, y, z, w \in F
$$

The map which sends

$$
\left[\begin{array}{lll}
x & y & z \\
0 & x & w \\
0 & 0 & x
\end{array}\right] \text { to }\left[\begin{array}{lll}
x & w & z \\
0 & x & y \\
0 & 0 & x
\end{array}\right]
$$

is an involution ${ }^{*}$ on $R$. It is easy to verify that $\left(R,{ }^{*}\right)$ is a SPP-ring. It is not normal because for

$$
a=\left[\begin{array}{lll}
0 & 1 & 0 \\
0 & 0 & 0 \\
0 & 0 & 0
\end{array}\right]
$$

$a a^{*} \neq a^{*} a$.

LemMa 2. Let $\left(R,{ }^{*}\right)$ be a normal ring which is not commutative. Then for all $a, b \in R$

$$
T(a)=T(b), \quad N(a)=N(b)
$$

imply $x a=b x$ for some $x \in R, x \neq 0$.

Proof. First assume $b \neq a^{*}$. Since $T(a)=T(b), X=b-a^{*}=a-b^{*} \neq 0$ and we have $x a=\left(b-a^{*}\right) a=b a-a^{*} a=b a-b b^{*}=b\left(a-b^{*}\right)=b x$.

Next assume $b=a^{*}$ and $a \notin Z$. Then $x=[a, y] \neq 0$ for some $y \in R, y \neq 0$. Whence, $x a=[a, y] a=[a, y a]=\left[a^{*}, a^{*} y^{*}\right]=a^{*}\left[a^{*}, y^{*}\right]=a^{*}[a, y]=b x$.

Lastly, assume $b=a^{*}$ and $a \in Z$. Since $R$ is not commutative, there exists non-zero elements $y, z$ in $R$ such that $x=[y, z] \neq 0$. Hence, $x a=[y, z] a=$ $[y, z a]=\left[y^{*}, a^{*} z^{*}\right]=a^{*}\left[y^{*}, z^{*}\right]=a^{*}[y, z]=b x$.

The converse to the above is not true in general.

ExAmple 2. Let $F$ be a field of char $\neq 2$ and $R$ be the ring of $2 \times 2$ matrices over $F$. The map * defined by

$$
\left[\begin{array}{cc}
t & u \\
v & w
\end{array}\right]^{*}=\left[\begin{array}{cc}
w & -u \\
-v & t
\end{array}\right], \quad t, u, v, w \in F
$$


is an involution on $R$. It is easily verified that $\left(R,{ }^{*}\right)$ is a normal ring. For

$$
a=\left[\begin{array}{ll}
1 & 0 \\
0 & 1
\end{array}\right], \quad b=x=\left[\begin{array}{ll}
1 & 0 \\
0 & 0
\end{array}\right],
$$

$x a=x=b x$ but clearly $a$ and $b$ have distinct traces and norms.

The converse, however, is true if $R$ has no zero divisors.

THEOREM. Let $\left(R,{ }^{*}\right)$ be a normal ring which is not commutative and has no zero divisors. Then for all $a, b \in R$,

$$
T(a)=T(b), \quad N(a)=N(b)
$$

if and only if

$$
x a=b x \text { for some } x \in R, \quad x \neq 0 .
$$

Proof. Assume $x a=b x$ for sime $x \in R, x \neq 0$. Then $x T(a) x^{*}=T\left(x a x^{*}\right)=$ $T\left(b x x^{*}\right)=T\left(b x^{*} x\right)=T\left(x b x^{*}\right)=x T(b) x^{*} \quad$ and $\quad x a a^{*} x^{*}=b x x^{*} b^{*}=b x^{*} x b^{*}=$ $b x^{*}\left(b x^{*}\right)^{*}=\left(b x^{*}\right)^{*} b x^{*}=x b^{*} b x^{*}=x b b^{*} x^{*}$. Hence,

$$
T(a)=T(b) \text { and } a a^{*}=b b^{*} .
$$

The converse is Lemma 2.

Acknowledgement. The author wishes to thank his Ph.D. supervisor Dr. D. Ž. Djoković of the University of Waterloo, Ontario for many helpful comments.

\section{REFERENCES}

1. F. J. Dyson, Quaternion Determinants, Helvetica Physica Acta, Vol. 45 (1972), 289-302.

2. G. Maxwell, Algebras of Normal Matrices, Pacific Jour. of Math., Vol. 43, No. 2 (1972). 421-428.

60 UNDERHILL DRIVE \# 410

Don Mills, OnTario M3A 2J7

CANADA 